\title{
The best is the enemy of the good - can research ethics learn from rationing?
}

Tony Hope Oxford University, Oxford

The "best interests" of the patient are widely seen as a cornerstone of medical practice. They are the explicit legal standard for medical care in the case of patients who lack capacity to consent to treatment.

The best interests of the individual patient have, of course, long been compromised for the sake of other potential patients-in infectious disease control for example. The question of the ethics of such compromise became a hot issue, for UK doctors, about fifteen years ago in the context of health care rationing. At that time the fact that there are insufficient resources to provide all patients, in all circumstances, with the best possible treatment became explicit. Faced with this uncomfortable truth, doctors tended to divide into two camps. Some wanted to play no role in decisions abou resource allocation. They continued to be guided by the single ethic of each individual patient's best interests. However, "best interests" could be interpreted as "best" given the treatments that were made available by those who determined the allocation of resources. Others took the opposite approach: since choices had to be made as to the best use of available resources, doctors ought to be playing a significant role in making these choices.

This polarisation has softened over time. Doctors have become more at ease with the idea that some treatments are not, given the overall resources, cost-effective, even if they are the best available. Clinical guidelines increasingly are influenced by cost-effectiveness. To provide the best health care overall, within the available resources, doctors aim to provide good care for each individual patient, but they do not always provide the best care.

\section{Research ethics}

Research ethics is under scrutiny, particularly in the light of current debates over the revision of the Declaration of Helsinki. The declaration states: "Concern for the interests of the subject must always prevail over the interests of science and society". This seems generally to be taken to imply, for example, that when a patient takes part in a clinical trial the treatment within that trial should be in his or her best interests. Such interests should never, on this view, be compromised for the sake of those patients in the future who might benefit from the trial results. Randomised controlled trials, for example comparing the effectiveness of two treatments, should only be undertaken if there is "equipoise", and should be stopped once the trial data upset such equipoise. Equipoise means there is insufficient evidence to consider one of the two treatments as superior to the other. In other words, it is not against the best interests of a patient in the trial to receive either one treatment or the other. Applying this criterion, clinical trials should typically be stopped when significance levels in favour of one treatment reach a value of $p$ less than about 0.05 . To continue the trial beyond this point would not normally be in the best interests of those in the trial. Advocates of large clinical trials (proceeding to significance levels typically of $\mathrm{p}$ less than 0.001 or beyond) could support such trials for several reasons. One important reason might be that the rule for stopping the trial should be one that in general would lead to the best outcome overall, taking the interests of both trial subjects and future patients into account, and giving them equal weight.

Consider two strategies. Two drugs, A and B, are to be compared. After the trial a very large number of people with the relevant disease will be treated with whichever of the drugs is found to be better. In strategy 1 the trial is stopped when the results show that there is no longer equipoise in the sense given above. In strategy 2 the trial is continued until a point where the results are much more significant (say p less than 0.001 , or even 0.0001 ). If we adopt strategy 1 and stop the trial relatively early there is a much higher chance that we make a mistake and conclude, correctly on the evidence, that $\mathrm{A}$ is better than B when in fact B is better than A. If the trial affects practice worldwide, and its results do lead to the wrong conclusion, an enormous number of patients will be worse off through getting the worse treatment. Thus adopting strategy 2 , with a very large trial and stringent significance requirements, might lead to fewer deaths overall.

\section{Trialasia and Equipoisia}

Consider two hypothetical countries, Trialasia and Equipoisia. The health care is identical in these two countries at time $t$, and is remarkably similar to the $\mathrm{UK}$ in the year 2000. In Trialasia, from time $\mathrm{t}$ 
onwards, clinical trials are continued to a point well beyond equipoise in many situations. In Equipoisia, clinical trials are stopped once there is no longer equipoise. The two countries are carefully observed. After three years, analysis shows that more people have died within trials in Trialasia but that fewer people have died outside trials. Overall there have been fewer medical deaths in Trialasia. In neither country do any patients receive worse treatment than the best treatment that was available until a year or so ago.

After ten years there has been slightly more rapid advance in medical therapies in Trialasia as a result of the more secure judgments that have been made as to which therapies are more effective. In both countries a trial is underway for the same specific clinical condition. In Trialasia drug $\mathrm{F}$ is being compared with drug $\mathrm{G}$. The trial has run well beyond equipoise. The evidence is very good that drug $\mathrm{G}$ is the more effective. Drug F was the drug of choice before this current trial. In fact a few years back drug $\mathrm{F}$ had been compared, in Trialasia, with drug $\mathrm{E}$, and the result of the large trial showed that drug $\mathrm{F}$ was superior. In Equipoisia the trial is comparing drug $\mathrm{E}$ with drug F. Drug $\mathrm{G}$ has not yet been developed in Equipoisia. Patients in the trial taking place in Equipoisia are receiving either drug $\mathrm{E}$ or $\mathrm{F}$. The patients in the trial in Trialasia are receiving either $\mathrm{F}$ or $\mathrm{G}$. Thus no patient in the trial being undertaken in Trialasia is receiving worse treatment than the best treatment being given in Equipoisia; and some patients in the trial in Trialasia are receiving better treatment than any patients in the trial (or in clinical practice) in Equipoisia. When the two countries are compared after a further ten years it is found that, as a result of the faster progress in treatment development in Trialasia, all patients, both in trials and outside trials, are receiving better treatment in Trialasia than any patients (whether in trials or not) in Equipoisia.

Good treatment, but not always the best

Pursuing clinical trials beyond equipoise, in order to ensure that the trial conclusions are almost certainly correct, can be beneficial in two related ways.
First it can be better for patients in the future who are more likely to receive the genuinely better treatment. Second, by establishing with high levels of certainty what treatments are best it is likely that therapeutic progress will be most rapid. Within a few years this will have the effect that everyone is better off, even those in trials, if we adopt the criteria for stopping trials used in Trialasia rather than in Equipoisia. Although, at any one time, some patients within clinical trials in Trialasia are not receiving the best available treatment, they are receiving what was the best treatment only a short while ago. But even this will be better than the treatments available in Equipoisia.

In the area of resource allocation we sometimes provide less than the best treatment for one patient in order not to disadvantage another patient excessively. Justice demands balancing the interests of different patients. Is it very different if we offer a patient now, in the context of a large clinical trial, a treatment that is not, on the evidence, the very best, on the grounds that this will benefit other patients in the future? Doesn't justice demand some curtailing of the clinical freedom of doctors and patients? Perhaps for the sake of future patients, some established treatments should only be available as part of a clinical trial. Clinical practice and clinical trials might develop so that much clinical practice is carried out in the context of well-designed and well-managed clinical trials. Whilst all patients will receive good treatment, it would be unfair on future patients if all current patients were necessarily to receive the very best treatments.

Tony Hope is Editorial Associate of the Journal of Medical Ethics and Professor of Medical Ethics, University of Oxford, UK.

\section{References}

1 World Medical Association. Declaration of Helsinki. Hong Kong: WMA, 1989 